\title{
Medical Image of the Week: Granulomatous Amoebic Encephalitis
}

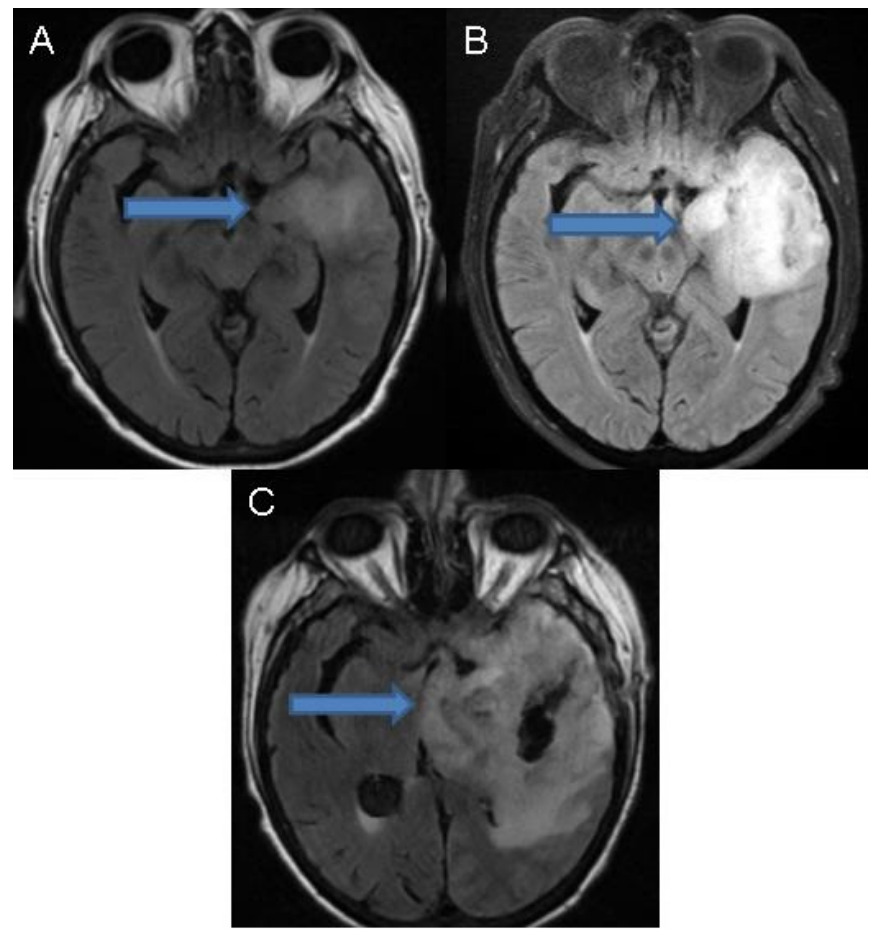

Figure 1. Progressively worsened encephalitis with increasing T2/FLAIR hyperintensity, with restricted diffusion and increasing cortical enhancement in the left anterior/medial temporal lobe and inferior frontal lobe, multifocal areas of hemorrhage, mass effect and interval development of multiple progressive areas of rim enhancement with small areas of restricted diffusion suggested new abscess formation.

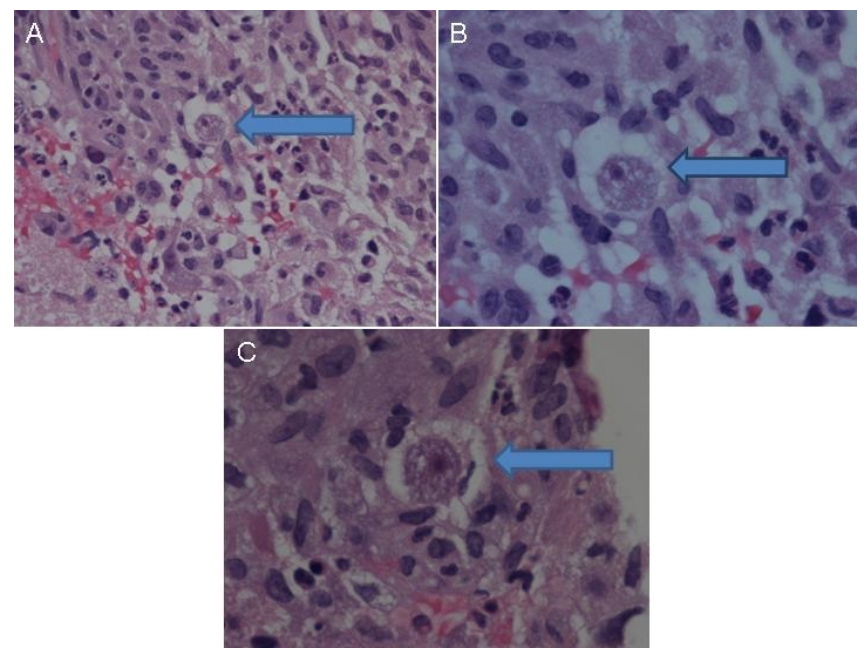

Figure 2. Necrotizing granulomas with acute inflammation and microorganisms with the morphologic features of amoeba (H \& E stained slides: 500X and 1000X). 
A 64-year-old woman with history of deceased donor kidney transplantation presented with altered mental status. MRI of the brain showed new region of T2/FLAIR hyperintensity with restricted diffusion and slight cortical enhancement in the left middle temporal lobe (Figure 1, Panel A). Her neurological exam was notable for expressive aphasia and right-sided weakness. Initial diagnosis of ischemic stroke was further evaluated due to immunosuppressive status. Her CSF showed a WBC of 12 cells/microL with $80 \%$ lymphocytes, glucose $61 \mathrm{mg} / \mathrm{dL}$, and protein $53 \mathrm{mg} / \mathrm{dL}$. Follow up MRI showed progression of T2/FLAIR hyperintensity, intraparenchymal hemorrhage, and peripheral patchy enhancement in the left anterior/medial temporal lobe and inferior frontal lobe suspicious for encephalitis (Figure 1, Panel B). Left temporal lobe biopsy revealed granulomatous encephalitis with microorganisms morphologically consistent with amoeba (Figure 2), and tissue cultures grew MRSA. Acanthamoeba species was confirmed by the Centers for Disease Control and Prevention (CDC) with antibody testing. Immunosuppression was tapered. She was treated with vancomycin and a CDC approved regimen of pentamidine, sulfadiazine, flucytosine, fluconazole, azithromycin, and miltefosine. Repeat MRI revealed continued progression of encephalitis with increased T2/FLAIR hyperintensity, mass effect, multifocal hemorrhage and new abscess formation (Figure 1, Panel C). Despite aggressive medical management, her neurologic status continued to deteriorate. Given her grim prognosis and failure to show clinical improvement, her family decided to pursue hospice care.

Granulomatous amebic encephalitis is a life-threatening central nervous system infection caused by the free-living amoebae Acanthamoeba spp., Balamuthia mandrillaris and Sappinia pedata. Onset is subacute to chronic affecting predominantly the immunocompromised population. The diagnosis requires high index of suspicion, and early diagnosis is crucial to survival. Radiological findings are nonspecific and can be seen in CNS tuberculosis, neurocysticercosis, disseminated encephalomyelitis, viral encephalitis etc. Multiple antibiotics targeting various proteins or receptors are required for successful treatment. A combination of surgical and medical interventions may be required to prevent morbidity and mortality.

Ateefa Chaudhury MD ${ }^{1}$, Christopher Geffre $\mathrm{MD}^{2}$, and Tauseef Afaq Siddiqi MD ${ }^{3}$

${ }^{1}$ Department of Medicine

${ }^{2}$ Department of Pathology

${ }^{3}$ Division of Pulmonary, Allergy, Critical Care and Sleep Medicine

The University of Arizona, Tucson, AZ

\section{Reference}

1. Parija SC, Dinoop K, Venugopal H. Management of granulomatous amebic encephalitis: Laboratory diagnosis and treatment. Trop Parasitol. 2015;5(1):23-8. [CrossRef] [PubMed] 